\title{
AN ALGORITHM FOR COMPLEMENTS OF FINITE SETS OF INTEGERS
}

\author{
GERALD WEINSTEIN
}

\begin{abstract}
Let $A_{k}=\left\{0, a_{2}, a_{3}, \ldots, a_{k}\right\}$ and $B=\left\{0, b_{2}, b_{3}, \ldots\right\}$ be sets of nonnegative integers of $k$ elements and infinitely many elements, respectively. Suppose $B$ has asymptotic density $x: d(B)=x$. If, for every integer $n \geqq 0$, we can find $a_{i} \in A_{k}, b_{j} \in B$ such that $n=a_{i}+b_{j}$, then we say that $A_{k}$ has a complement of density $\leqq x$.

Given $A_{k}$ and $x$ there is no known algorithm for determining if such a set $B$ exists.

We define regular complement and give an algorithm for determining if $B$ exists when complement is replaced by regular complement. More precisely, given $A_{4}$ and $x=1 / 3$ we give an algorithm for determining if $A_{4}$ has a regular complement $B$ with density $\leqq 1 / 3$. We relate this result to the

Conjecture. Every $A_{4}$ has a complement of density $\leqq 1 / 3$.
\end{abstract}

Let $A_{k}=\left\{0, a_{2}, a_{3}, \ldots, a_{k}\right\}$ and $B=\left\{0, b_{2}, b_{3}, \ldots, b_{n}, \ldots\right\}$ be sets of nonnegative integers of $k$ elements and infinitely many elements, respectively. If, for every integer $n \geqq 0$, we can find $a_{i} \in A_{k}, b_{j} \in B$ such that $n$ $=a_{i}+b_{j}$, then $B$ is said to be a complement of $A$.

Let $B(n)$ be the number of elements in $B$ which are $\leqq n$, and define $d(B)$, the density of $B$, as follows:

$$
d(B)=\lim _{n \rightarrow \infty} B(n) / n \quad \text { if this limit exists. }
$$

From now on we consider only those sets $B$ for which the density exists.

For a given set $A_{k}$ we wish to find the complementary set $B$ with minimum density. More precisely, we define $c\left(A_{k}\right)$, the codensity of $A_{k}$, as follows:

$$
c\left(A_{k}\right)=\inf _{B} d(B) \quad \text { where } B \text { ranges over all complements of } A_{k} .
$$

Finally we define $c_{k}$ as the "largest" codensity of any $A_{k}$. More precisely,

$$
c_{k}=\sup _{A_{k}} c\left(A_{k}\right)
$$

D. J. Newman proved [1] that $c_{3}=2 / 5$ and also that $c_{k} \sim(\log k) / k$. We proved [2] that $1 / 3 \leqq c_{4}<.339934$.

Given a set $A_{k}$ suppose we can find a set $B=\left\{b_{1}, b_{2}, \ldots, b_{n}\right\}$ and a number

Received by the editors January 8, 1975.

AMS (MOS) subject classifications (1970). Primary 10L10. 
$N$ such that $A_{k} \oplus B \equiv[0,1,2, \ldots, N-1](\bmod N)$. Then the codensity of $A_{k}$ is $\leqq n / N$.

In [2] we introduced the concept of regular complement. If, in the previous paragraph, the complement $B$ consists entirely of consecutive multiples of a given element, i.e., $B=\{M, 2 M, 3 M, \ldots, n M\}$, then we say that $A_{k}$ has a regular complement of density $\leqq n / N$.

Not only is there no known algorithm for determining the codensity of $A_{k}$, there is not even one for determining whether $A_{k}$ has a complement $B$ such that $d(B) \leqq x$. It is the purpose of this note to remedy the situation somewhat by giving an algorithm for answering the question: Does $A_{k}$ have a regular complement of density $\leqq x$ ? We actually give a method for determining whether $A_{4}$ has a regular complement of density $\leqq 1 / 3$, because of its obvious application to the

Conjecture. $c_{4}=1 / 3$.

However, the generalization of our result presents no difficulties.

We adopt the following conventions throughout: $A_{4}$ represents a set of four integers, $A_{4}=\left\{a_{1}, a_{2}, a_{3}, a_{4}\right\}$, with $0=a_{1}<a_{2}<a_{3}<a_{4} . M$ and $N$ are positive integers, with $M<N$ and $(M, N)=1 . i, j, k, l$ is a permutation of 1 , $2,3,4$.

LEMMA 1. Given $A_{4}$ and $B=\{M, 2 M, \ldots,[N / 3] M\}$, consider $a$ set of the form $D=\left\{a_{i}-a_{j}, a_{j}-a_{k}, a_{k}-a_{l}, a_{l}-a_{i}\right\}(\bmod N)$. Then $B$ is a regular complement of $A$ if and only if $D \subset B$ for some permutation of $i, j, k, l$.

Proof. We write $a_{i}=K_{i} M, a_{j}=K_{j} M, a_{k}=K_{k} M, a_{l}=K_{l} M\left(K_{n}<N, n\right.$ $=1, \ldots, 4)$. Assume that $D \subset B$. Since $\left\{K_{i}-K_{j}, K_{j}-K_{k}, K_{k}-K_{l}, K_{l}\right.$ $\left.-K_{i}\right\}(\bmod N) \subset\{1,2, \ldots,[N / 3]\}$, it must be true that no two adjacent $K^{\prime} s$ are separated by a gap larger than $[N / 3]$. Therefore, since $\{1,2, \ldots,[N / 3]\}$ is a set of $[N / 3]$ consecutive numbers, it is a complement to $\left\{K_{i}, K_{j}, K_{k}, K_{l}\right\}$. Hence, $\{M, 2 M, \ldots,[N / 3] M\}$ is a complement to $\left\{K_{i} M, K_{i} M, K_{k} M, K_{l} M\right\}$. Now assume that $B$ is a complement to $A$. It follows that $\{1,2, \ldots,[N / 3]\}$ is a complement to $\left\{K_{i}, K_{j}, K_{k}, K_{l}\right\}$ and therefore the gap between adjacent $K^{\prime} \mathrm{s}$ is $\leqq[N / 3]$. We assume, without loss of generality, that $K_{l}<K_{k}<K_{j}<K_{i}$, and so $D \subset B$.

LEMMA 2. Given $A_{4}$ consider the congruences:

$$
\begin{aligned}
k_{1} M & \equiv a_{i}-a_{j} \\
k_{2} M & \equiv a_{j}-a_{k} \\
k_{3} M & \equiv a_{k}-a_{l} \\
k_{4} M & \equiv a_{l}-a_{i}
\end{aligned}
$$

Then $A_{4}$ has a regular complement of density $\leqq[N / 3]$ if and only if there exists a solution of (1) with $k_{i} \leqq[N / 3], i=1, \ldots, 4$.

Proof. If $A_{4}$ has a regular complement of density $\leqq[N / 3]$ then, by Lemma $1, \quad\left\{a_{i}-a_{j}, a_{j}-a_{k}, a_{k}-a_{l}, a_{l}-a_{i}\right\} \subset\{M, 2 M, \ldots,[N / 3] M\}(\bmod N) . \quad$ If 
there exists a solution of the required type then, by Lemma $1, A_{4}$ has a regular complement of density $\leqq[N / 3]$. have

Define $k_{0}$ by $k_{0} M \equiv 1(\bmod N)$ and let $r=k_{0} / N$. Let $d_{i j}=\left|a_{i}-a_{j}\right|$. We

LemMa 3. Let $i>j$ imply $a_{i}>a_{j}$. The congruence

$$
K M \equiv a_{i}-a_{j}(\bmod N)
$$

has a solution $K \leqq[N / 3]$ if and only if $r$ satisfies one of the inequalities:

$$
\begin{aligned}
& \frac{3(k-1)}{3 d_{i j}}<r \leqq \frac{3(k-1)+1}{3 d_{i j}} \text { if } i>j, \\
& \frac{3 k-1}{3 d_{i j}} \leqq r<\frac{3 k}{3 d_{i j}} \quad \text { if } i<j,
\end{aligned}
$$

Proof. Suppose $K \leqq[N / 3]$. If $i>j$, then $K M \equiv a_{i}-a_{j}(\bmod N)$ implies $K M$ $\equiv d_{i j}(\bmod N)$. However, since $k_{0} M \equiv 1(\bmod N)$, we have $d_{i j} k_{0} M \equiv d_{i j}(\bmod N)$ so that $K \equiv d_{i j} k_{0}(\bmod N)$. Therefore, $d_{i j} r \equiv s(\bmod 1)$ where $0<s \leqq 1 / 3$. This implies that

$$
\frac{3(k-1)}{3}<d_{i j} r \leqq \frac{3(k-1)+1}{3} \text { or } \frac{3(k-1)}{3 d_{i j}}<r \leqq \frac{3(k-1)+1}{3 d_{i j}}
$$

for some $k, 1 \leqq k \leqq d_{i j}$. If $i<j$, then $K \equiv-d_{i j} k_{0}(\bmod N)$ and so $-d_{i j} r$ $\equiv s(\bmod 1), 0<s \leqq 1 / 3$ implies

$$
d_{i j} r \equiv-s(\bmod 1) \quad \text { or } \quad d_{i j} r \equiv t(\bmod 1), \quad 2 / 3 \leqq t<1 .
$$

This implies that

$$
\frac{3 k-1}{3} \leqq d_{i j} r<\frac{3 k}{3} \text { or } \frac{3 k-1}{3 d_{i j}} \leqq r<\frac{3 k}{3 d_{i j}}
$$

for some $k, 1 \leqq k \leqq d_{i j}$. Since the argument can also be read backwards, for $i>j$ and $i<j$, this completes the proof.

As we showed in Lemma 2, the existence of a regular complement of density $\leqq[N / 3]$ is guaranteed if there exists a solution to the congruences (1) for some permutation of $i, j, k, l$. We will show that not all six permutations need be examined -three are sufficient.

First we note that $D \subset B$, in Lemma 1 , and the solvability of the congruences (1), in Lemma 2 , are both equivalent to the following. If we list the numbers $M, 2 M, 3 M, \ldots,(N-1) M, N M \equiv 0$, then the numbers 0 $=a_{1}, a_{2}, a_{3}, a_{4}$ occur in this list in some order, say $a_{2}, a_{3}, a_{4}, a_{1}$, in such a way that adjacent $a_{i}$ 's are separated by fewer than $[N / 3]$ numbers.

LEMMA 4. The only congruences one need attempt to solve, in Lemma 1, are those involving the following orderings of the $a_{i}$ 's:

$$
\begin{array}{llll}
a_{2} & a_{3} & a_{4} & a_{1},
\end{array}
$$




$$
\begin{array}{llll}
a_{3} & a_{2} & a_{4} & a_{1}, \\
a_{2} & a_{4} & a_{3} & a_{1} .
\end{array}
$$

Proof. Since all orderings end with $a_{1}=0$, there are only six permutations to consider. The remaining three are:

$$
\begin{array}{llll}
a_{4} & a_{3} & a_{2} & a_{1}, \\
a_{4} & a_{2} & a_{3} & a_{1}, \\
a_{3} & a_{4} & a_{2} & a_{1} .
\end{array}
$$

We will show that if a primed ordering occurs, then so does the respective unprimed ordering. Clearly one is the reverse of the other. If a primed ordering occurs in the set of numbers $M, 2 M, \ldots,(N-1) M, N M$, then we obtain the same numbers in reverse order: $(N-M), 2(N-M), \ldots,(N-1)(N-M)$, $N(N-M)$. Hence we obtain the unprimed ordering.

Recalling that $d_{i j}=\left|a_{i}-a_{j}\right|$, Lemma 4 indicates that there are three sets of differences which are of interest to us:

Case I. $d_{43}, d_{32}, d_{21}, d_{14}$.

Case II. $d_{42}, d_{23}, d_{31}, d_{14}$.

Case III. $D_{34}, d_{42}, d_{21}, d_{13}$.

Each difference $d_{i j}$ determines a set of intervals $R_{i j}$ on the unit interval:

$$
R_{i j}=\left\{\begin{array}{ll}
\left(\frac{3(k-1)}{3 d_{i j}}, \frac{3(k-1)+1}{3 d_{i j}}\right] & \text { if } i>j, \\
{\left[\frac{3 k-1}{3 d_{i j}}, \frac{3 k}{3 d_{i j}}\right)} & \text { if } i<j,
\end{array} \quad k=1,2, \ldots, d_{i j}\right\} .
$$

Our result can now be expressed in the following

THEOREM. $A_{4}$ does not have a regular complement of density $\leqq 1 / 3$ if and only if

$$
\begin{aligned}
& R_{43} \cap R_{32} \cap R_{21} \cap R_{14}=\varnothing, \\
& R_{42} \cap R_{23} \cap R_{31} \cap R_{14}=\varnothing, \\
& R_{34} \cap R_{42} \cap R_{21} \cap R_{13}=\varnothing .
\end{aligned}
$$

Proof. It is clear from Lemma 3 that for the congruences

$$
\begin{aligned}
k_{1} M & \equiv a_{4}-a_{3} \\
k_{2} M & \equiv a_{3}-a_{2} \\
k_{3} M & \equiv a_{2}-a_{1} \\
k_{4} M & \equiv a_{1}-a_{4}
\end{aligned}
$$

to be solved simultaneously in $M$ and $N$, with $k_{i} \leqq[N / 3], i=1, \ldots, 4$, it is necessary and sufficient that $R_{43} \cap R_{32} \cap R_{21} \cap R_{14} \neq \varnothing$. From Lemma 4 
we see that only three sets of congruences are involved-hence the equations (3), (4), and (5).

There do exist sets $A_{4}$ which do not have regular complements of density $\leqq 1 / 3$. A computer search has revealed that for $A_{4}$, with $a_{4} \leqq 100$, only two such sets exist! They are $\{0,1,7,9\}$ and $\{0,3,5,11\} .^{1}$ Both these sets have complements of density $\leqq 1 / 3$.

We conjecture that at most a finite number of sets $A_{4}$ do not have regular complements of density $\leqq 1 / 3$. We have attempted to prove this by assuming that $a_{4}$ is large and that equations (3), (4), and (5) are satisfied. So far we have failed to find the desired contradiction.

ACKNOWLEDGEMENT. This paper is part of the author's doctoral dissertation completed at Yeshiva University under the direction of Professor D. J. Newman. The author expresses his appreciation to Professor Newman for his advice and encouragement during the preparation of this paper.

\section{REFERENCES}

1. D. J. Newman, Complements of finite sets of integers, Michigan Math. J. 14 (1967), 481-486. MR 36 \# 1411.

2. G. Weinstein, Some covering and packing results in number theory, J. Number Theory (to appear).

Department of Mathematics, City College of New York, New York, New York 10031

$1\{0,2,8,9\}$ and $\{0,6,8,11\}$ also turned up, but the first has the same difference set as $\{0,1,7,9\}$ and the second has the same difference set as $\{0,3,5,11\}$. Hence, more strictly speaking, only two equivalence classes of sets exist which do not have regular complements for $a_{4} \leqq 100$. 